\title{
Keine zusätzlichen antidepressiven Effekte durch Ketamin
}

Fragestellung: Sind Elektrokonvulsionstherapien (EKT) besser wirksam, wenn Ketamin als Add-on-Therapie oder als alternatives Anästhetikum eingesetzt wird?

Hintergrund: Ketamin wird aktuell sehr intensiv bezüglich seiner antidepressiven Eigenschaften untersucht, und neue Studien weisen darauf hin, dass die akuten antidepressiven Effekte sehr vielversprechend sind. Insofern liegt die Frage nahe, ob der Effekt einer EKT durch Ketamin verstärkt werden kann. Die Autoren gingen dieser Frage in einem systematischen Review mit Metaanalyse nach.

Patienten und Methodik: Die Autoren führten ein Update einer früheren Metaanalyse durch [1]. Sie suchten in drei großen Datenbanken nach randomisierten kontrollierten Studien mit EKT und Ketamin bis einschließlich 30. Juli 2016. Die Metaanalyse wurde nach den PRISMA-Leitlinien durchgeführt. Hauptzielparameter war die Veränderung in den Depressionswerten auf der Hamilton-

McGirr A, Berlim MT, Bond DJ et al. Adjunctive ketamine in electroconvulsive therapy: updated systematic review and meta-analysis. Brit J Psychiatry 2017; 210: $403-7$
Depressions-Skala und der Montgomery Åsberg-Depressions-Skala, sekundäre Outcomes die Responserate (>50\% Reduktion depressiver Symptome) und die Ergebnisse kognitiver Tests.
Ergebnisse: Zehn randomisierte kontrollierte Studien wurden in die Metaanalyse eingeschlossen. Insgesamt wurden 602 depressive Patienten behandelt, wobei 333 Patienten eine (Addon-)Behandlung mit Ketamin erhielten und 269 Patienten in einer Kontrollgruppe mit Standard-EKT behandelt wurden. In allen Studien wurde Ketamin intravenös gegeben. Die applizierte Dosis schwankte zwischen 0,3 und $1-2 \mathrm{mg} / \mathrm{kg}$ Körpergewicht. Die Anzahl der EKT-Sitzungen betrug meist sechs bis acht, in einer Studie neun und in einer anderen Studie elf Sitzungen.

Für eine Add-on-Therapie mit Ketamin fand sich eine nicht signifikante Effektstärke von 0,18 bezüglich der Reduktion depressiver Symptome. Auch wenn alle sieben Studien ausgeschlossen wurden, bei denen ein Barbiturat zur Anästhesie verwendet wurde, blieb die Effektstärke nicht signifikant bei 0,24. Bezüglich der Response- und Remissionsraten fanden sich ebenfalls keine Vorteile einer Ketamin(Add-on)-Behandlung. Die Auswirkungen auf kognitive Funktionen waren sehr heterogen, eine Studie fand eine längere Zeit bis zur Reorientierung nach EKT. Hinweise für pro-kognitive Effekte fanden sich nicht.

Schlussfolgerungen: Die bisher durchgeführten Studien zeigen keinen besseren Nutzen einer EKT-Behandlung, wenn Ketamin zusätzlich oder als Alternative zu einem anderen Anästhetikum verwendet wird.

\section{Noch zu kleine Fallzahlen lassen sichere Aussagen nicht zu}

In die zehn Studien wurden nur relativ kleine Patientenzahlen eingeschlossen, sodass die kleinen Effektstärken von 0,18 beziehungsweise 0,24 nicht signifikant wurden. Insgesamt muss man davon ausgehen, dass erst ab einer behandelten Zahl von

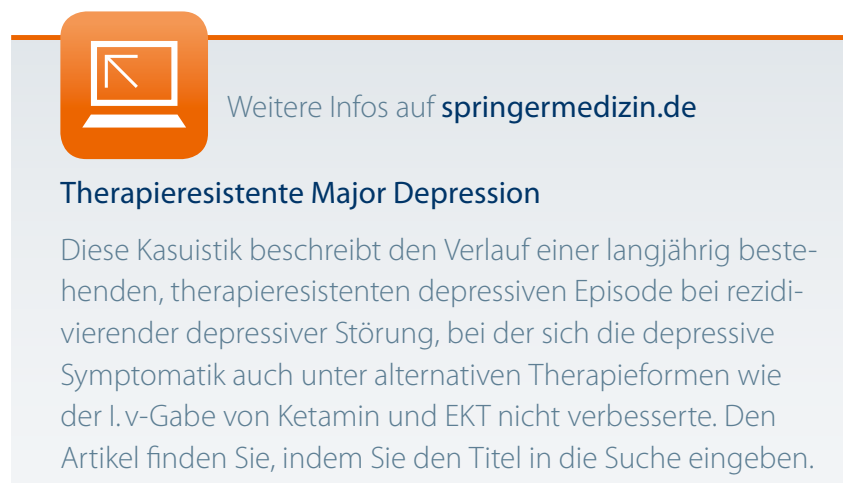

1.000 oder besser 2.000 Patienten sichere Effektstärken abgeschätzt werden können. Daher ist die Diskussion, ob Ketamin einen Zusatznutzen bei der EKT hat oder nicht, noch nicht abgeschlossen. Weitere Studien müssen durchgeführt werden. Bestätigen sich die kleinen Effektstärken, wäre auch dieser kleine Effekt klinisch relevant, da es bisher keine etablierten Augmentierungsverfahren bei der EKT gibt.

Bemerkenswert ist, dass die Autoren einen Zusammenhang zwischen Studienqualität und Ergebnissen fanden. Die Studien mit hoher methodischer Qualität fanden durchgehend keinen Effekt einer Zusatzbehandlung mit Ketamin. Um sichere Aussagen treffen zu können, wird es daher wichtig sein, dass die weiteren vorgelegten Studien eine hohe Qualität aufweisen.

\footnotetext{
Referenz

1. McGirr A et al. J Psychiatry Res $2015 ; 62: 23-30$
} 